\title{
A KINETIC APPROACH TO GENERAL FIRST ORDER QUASILINEAR EQUATIONS
}

\author{
BY \\ YOSHIKAZU GIGA ${ }^{1}$, TETSURO MIYAKAWA ${ }^{2}$ AND SHINNOSUKE OHARU ${ }^{2}$
}

\begin{abstract}
This paper presents a new method for constructing entropy solutions of first order quasilinear equations of conservation type, which is illustrated in terms of the kinetic theory of gases. Regarding a quasilinear equation as a model of macroscopic conservation laws in gas dynamics, we introduce as the corresponding microscopic model an auxiliary linear equation involving a real parameter $\xi$ which plays the role of the velocity argument. Approximate solutions for the quasilinear equation are then obtained by integrating solutions of the linear equation with respect to the parameter $\xi$. All of these equations are treated in the Fréchet space $L_{\text {loc }}^{1}\left(R^{n}\right)$, and a convergence theorem for such approximate solutions to the entropy solutions is established with the aid of nonlinear semigroup theory.
\end{abstract}

Introduction. The purpose of this paper is to present a new method for constructing global weak solutions of the Cauchy problem for the first order quasilinear equation of conservation type with variable coefficients:

$$
\begin{gathered}
u_{t}+\sum_{i=1}^{n} A^{i}(x, u)_{x_{i}}+B(x, u)=0 \quad\left(x \in R^{n}, t>0\right), \\
u(x, 0)=u_{0}(x) .
\end{gathered}
$$

Using the vanishing viscosity method under suitable regularity and boundedness conditions on $A^{i}$ and $B$, Kružkov [10] proved that for every bounded measurable initial function $u_{0}$ there exists a function $u$ on $R^{n} \times[0, \infty)$ which is a solution of $(\mathrm{M})$ in the following sense:

(i) $u$ is in $L^{\infty}\left(R^{n} \times(0, T)\right)$ for every $T>0$;

(ii) $u(\cdot, t) \rightarrow u_{0}$ in $L_{\text {loc }}^{1}\left(R^{n}\right)$ as $t \rightarrow 0$; and

(iii) for every $k \in R^{1}$ and every nonnegative function $\phi \in C_{0}^{\infty}\left(R^{n} \times(0, \infty)\right)$, the following inequality holds:

$$
\begin{gathered}
\int_{0}^{\infty} \int \phi_{t}|u-k| d x d t+\int_{0}^{\infty} \int \operatorname{sgn}(u-k) \sum_{i=1}^{n}\left(A^{i}(x, u)-A^{i}(x, k)\right) \phi_{x_{i}} d x d t \\
-\int_{0}^{\infty} \int \operatorname{sgn}(u-k) \phi\left[\sum_{i=1}^{n} A_{x_{i}}^{i}(x, k)+B(x, u)\right] d x d t \geq 0,
\end{gathered}
$$

Received by the editors November 28, 1983.

1980 Mathematics Subject Classification. Primary 35L60, 35Q20, 47H20.

Key words and phrases. First order quasilinear equations, conservation laws, entropy condition, nonlinear semigroups.

${ }^{1}$ Partially supported by The Sakkokai Foundation.

${ }^{2}$ Partially supported by Grant-in-Aid for Scientific Research, the Japan Ministry of Education, Science and Culture. 
where $\operatorname{sgn}(y)=y /|y|$ if $y \neq 0$; and $\operatorname{sgn}(0)=0$. It is easily seen from (E) that $u$ is a weak solution of $(M)$, i.e., it satisfies

$$
\int_{0}^{\infty} \int\left[u \phi_{t}+\sum_{i=1}^{n} A^{i}(x, u) \phi_{x_{i}}-B(x, u) \phi\right] d x d t=0
$$

for $\phi \in C_{0}^{\infty}\left(R^{n} \times(0, \infty)\right)$. When $A^{i}$ and $B$ are nonlinear in $u$, the uniqueness of generalized solutions is not always ensured in the class of weak solutions as mentioned above; see [11]. However, it is shown in [10] that given a bounded function $u_{0}$ there exists exactly one weak solution of (M) satisfying (i)-(iii). In what follows, a solution of (M) satisfying (i)-(iii) will be called an entropy solution since (iii) is regarded as a generalization of the entropy condition in the sense of Oleĭnik [15].

In this paper we discuss the construction of entropy solutions of $(\mathrm{M})$ by applying a method evolved in the previous work [5] of the first two authors. To explain our method we recall two ways for describing the motion of gases: the macroscopic and microscopic approaches. The so-called Boltzmann equation governs the evolution in time of the microscopic state of gases, while the conservation laws in fluid mechanics describe the macroscopic thermo-fluid properties of gases. Both descriptions are connected in the following way: Given a microscopic quantity on the phase space, the corresponding macroscopic quantity is given by integrating the microscopic quantity with respect to the velocity argument. We take this point of view to construct approximate solutions of the Cauchy problem (M). Namely, we regard (M) as a model of macroscopic conservation laws in fluid mechanics and then formulate the corresponding microscopic model as the linear problem:

$$
f_{t}+\sum_{i=1}^{n}\left(a^{i}(x, \xi) f\right)_{x_{i}}+b(x, \xi) f=c(x, \xi), \quad f(x, \xi, 0)=f_{0}(x, \xi)
$$

where $f=f(x, \xi, t), a^{i}(x, \xi)=A_{\xi}^{i}(x, \xi)$ and $b(x, \xi)=B_{\xi}(x, \xi)$. The parameter $\xi \in R^{1}$ plays the role of the velocity argument as in the kinetic theory of gases. The macroscopic quantity corresponding to $f$ is then defined by the integral

$$
v(x, t)=\int_{-\infty}^{\infty} f(x, \xi, t) d \xi
$$

We then introduce (in $\S 1$ ) a function $F(w, \xi)$ of two real variables so that every macroscopic quantity $w$ is decomposed as

$$
w=\int_{-\infty}^{\infty} F(w, \xi) d \xi
$$

If we set $f_{0}(x, \xi)=F\left(u_{0}(x), \xi\right)$, then it is to be expected that the function $v$ approximates the solution of $(\mathrm{M})$. To ensure this we impose the following compatibility conditions:

$$
\begin{aligned}
& A^{i}(x, w)-A^{i}(x, 0)=\int_{-\infty}^{\infty} a^{i}(x, \xi) F(w, \xi) d \xi \quad(i=1, \ldots, n) \\
& B(x, w)-B(x, 0)=\int_{-\infty}^{\infty} b(x, \xi) F(w, \xi) d \xi \\
& c(x, \xi)=F(C(x), \xi), \quad C(x)=-\sum_{i=1}^{n} A_{x_{i}}^{i}(x, 0)-B(x, 0) .
\end{aligned}
$$


Indeed, conditions (C) and (D) imply that $v$ satisfies (M) at $t=0$. This suggests that for small positive $h$ approximate solutions can be successively constructed to satisfy $(\mathrm{M})$ for $t=j h, j=0,1, \ldots$, and that the aimed solutions of $(\mathrm{M})$ are obtained as the limits as $h \downarrow 0$ of such approximate solutions.

In [5] the method described above was employed to treat the special case $A^{i}=$ $A^{i}(u), B=0$, though the uniqueness problem of the constructed solutions was not discussed in detail. Our result not only extends the previous one to the case of more general equations, but also shows that the weak solutions constructed in [5] and in this paper are entropy solutions. The uniqueness result in the above-mentioned special case was also given independently by Kobayashi [8] by the systematic use of nonlinear semigroup theory in the Banach space $L^{1}\left(R^{n}\right)$. It is possible to extend our result to a more general case in which $A^{i}=A^{i}(x, t, u)$ and $B=B(x, t, u)$. Some of the results in this direction will be discussed in the forthcoming paper [18].

Features. For a review of the standard approaches to (M) that are mainly based on the vanishing viscosity method, we refer, for example, to $[\mathbf{3}, \mathbf{4}, 14$ and 15]. In contrast to these approaches our method is illustrated in terms of the kinetic theory of gases. The idea of using linear equations of the form $(\mathrm{m})$ to construct solutions of the original nonlinear equations is due to $\mathrm{S}$. Kaniel. He introduced a kinetic model for monatomic gases and made an attempt at the approximation of solutions of the Navier-Stokes equations. A similar approach to specific systems of conservation laws has been proposed by Harten, Lax and van Leer [7].

Our argument contains three features. Firstly, we are concerned with quasilinear equations involving both variable coefficients $A^{i}(x, u)$ and a lower order term $B(x, u)$ that can also be regarded as a forcing term. In order to deal with such a general form we need precise estimates for the solutions of the linear first order equation $(\mathrm{m})$ with variable coefficients. Secondly, we treat equations $(M)$ and $(\mathrm{m})$ in $L^{\infty}\left(R^{n}\right)$, which is viewed as a subspace of $L_{\text {loc }}^{1}\left(R^{n}\right)$. We show that approximate solutions constructed in $L^{\infty}\left(R^{n}\right)$ converge in the Fréchet space $L_{\text {loc }}^{1}\left(R^{n}\right)$ to the weak solutions of $(\mathrm{M})$. This approach enables us to construct entropy solutions associated with all initial functions bounded and measurable on $R^{n}$. Thirdly, we apply a theory of nonlinear semigroups in Banach space in order to derive various stability properties of the approximate solutions and to establish a convergence theorem for approximate solutions to entropy solutions. To apply this theory we introduce a family of appropriate weight functions, thereby imbedding $L^{\infty}$-bounded absolutely convex subsets of $L_{\text {loc }}^{1}\left(R^{n}\right)$ into the Banach space $L^{1}\left(R^{n}\right)$; and a convergence argument for approximate solutions lying in a fixed $L^{\infty}$-bounded set is passed through the weight functions to that in $L^{1}\left(R^{n}\right)$.

After this work was completed, our attention was called to the papers of $Y$. Brenier $[\mathbf{1 6}, \mathbf{1 7}]$. In $[\mathbf{1 7}]$ he deals with the case of $A^{i}=A^{i}(u)$ and $B=0$, and approximate solutions are constructed in the same way as in [5]. In [16] results are announced for the case in which $A^{i}=A^{i}(x, u)$ and $B=0$. All of these equations are considered in the subspace $L^{1}\left(R^{n}\right) \cap L^{\infty}\left(R^{n}\right)$ and the verification of the convergence of approximate solutions to entropy solutions is given by applying the method of Crandall and Majda [4]. It would be of interest to compare his approach with ours.

1. Statement of results. In the linear problem $(\mathrm{m})$ we put

$$
a^{i}(x, \xi)=A_{\xi}^{i}(x, \xi) \quad(i=1, \ldots, n), \quad b(x, \xi)=B_{\xi}(x, \xi),
$$


and impose the following conditions:

(H.1) For each $r>0, a^{i}, a_{x_{j}}^{i}, a_{x_{j} x_{k}}^{i}$ and $b, b_{x_{j}}, i, j, k=1, \ldots, n$, are bounded and continuous on $R^{n} \times[-r, r]$.

(H.2) $C(x) \equiv-\sum_{i=1}^{n} A_{x_{i}}^{i}(x, 0)-B(x, 0)$ and $C_{x_{j}}, j=1, \ldots, n$, are bounded and continuous on $R^{n}$.

(H.3) There are constants $\alpha, \beta \geq 0$ such that

$$
\alpha \geq-\sum_{i=1}^{n} a_{x_{i}}^{i}(x, \xi)-b(x, \xi), \quad \beta \geq-b(x, \xi) \quad \text { for all }(x, \xi) \in R^{n} \times R^{1} .
$$

Let $F(w, \xi)$ be the function defined by

$$
F(w, \xi)=\left\{\begin{array}{cl}
1 & \text { for } 0<\xi \leq w \\
-1 & \text { for } w \leq \xi<0 \\
0 & \text { otherwise }
\end{array}\right.
$$

Then $F$ satisfies conditions (C) and (D). Let $\left\{U_{\xi}(t) ; t \geq 0\right\}$ be the family of solution operators $U_{\xi}(t): L^{\infty}\left(R^{n}\right) \rightarrow L^{\infty}\left(R^{n}\right)$ of $(\mathrm{m})$ with $c=0$ and set

$$
V_{\xi}(t)=U_{\xi}(t) f_{0}+\int_{0}^{t} U_{\xi}(t-\sigma) c(\cdot, \xi) d \sigma,
$$

integrating in the sense of Bochner. According to the definition of the macroscopic quantity (I), we introduce a family of nonlinear operators $\left\{S_{t}: t \geq 0\right\}$ in $L^{\infty}\left(R^{n}\right)$ defined by

$$
S_{t} v=\int_{-\infty}^{\infty} V_{\xi}(t) f_{0} d \xi, \quad f_{0}(x, \xi)=F(v(x), \xi) \text { for } v \in L^{\infty}\left(R^{n}\right) .
$$

We then define for each $h>0$ an approximate solution $u^{h}$ of (M) by

$$
u^{h}(x, t)=\left(S_{t-h[t / h]} S_{h}^{[t / h]} u_{0}\right)(x),
$$

where $[a]$ denotes the greatest integer in $a \in R^{1}$. By definition $u^{h}$ satisfies (at least formally) equation (M) at $t=j h, j=0,1, \ldots$, so it is expected that $u^{h}$ will tend, as $h \rightarrow 0$, to a solution of (M) with initial value $u_{0}$. Indeed, we obtain the following theorem, which is the main result of this paper.

THEOREM I (CONVERGENCE THEOREM). Assume that conditions (H.1)(H.3) hold. Then given a $u_{0} \in L^{\infty}\left(R^{n}\right)$ the approximate solution $u^{h}$ defined by (1.1) converges to the entropy solution $u$ of $(\mathrm{M})$ with initial value $u_{0}$ and the convergence

$$
\lim _{h \rightarrow 0} u^{h}(\cdot, t)=u(\cdot, t) \quad \text { in } L_{\text {loc }}^{1}\left(R^{n}\right)
$$

holds uniformly for $t$ in bounded subintervals of $[0, \infty)$.

The definition of $F$ seems to be somewhat artificial. But it will be seen that $F$ is essentially the only function for which conditions (C) and (D) hold and the family $\left\{u^{h}\right\}$ of approximate solutions converges to the entropy solution of (M). The above procedure of constructing $u^{h}$ is explained in terms of the kinetic theory of gases in the following way: The operators $U_{\xi}(t)$ describe the free motion of gas particles with velocity $\xi$, while the substitution $v \rightarrow F(v, \xi)$ corresponds to collision 
processes. Further, letting $h \rightarrow 0$ in (1.1) is understood to be an analogy of the passage of the mean free path to zero.

Theorem I involves an existence theorem for entropy solutions with initial data in $L^{\infty}\left(R^{n}\right)$. Moreover, the convergence result (1.2) provides us with a new type of product formula for a nonlinear semigroup in the space $L_{\text {loc }}^{1}\left(R^{n}\right)$ associated with problem (M). By a semigroup of (nonlinear) operators on $L^{\infty}\left(R^{n}\right)$ we mean a oneparameter family $\{\mathfrak{S}(t): t \geq 0\}$ of (nonlinear) operators from $L^{\infty}\left(R^{n}\right)$ into itself satisfying the following conditions:

$(1.3) \mathfrak{S}(0)$ is the identity operator $\mathfrak{I}$ and $\mathfrak{S}(t+s)=\mathfrak{S}(t) \mathfrak{S}(s)$ on $L^{\infty}\left(R^{n}\right)$ for $s, t \geq 0$.

(1.4) For each $T>0$ and $r>0,\{\mathfrak{S}(t): 0 \leq t \leq T\}$ is equicontinuous on the $L^{\infty}$-ball $\left\{v \in L^{\infty}\left(R^{n}\right):\|v\|_{\infty} \leq r\right\}$ with respect to the metric topology of $L_{\text {loc }}^{1}\left(R^{n}\right)$.

(1.5) For each $v \in L^{\infty}\left(R^{n}\right), \mathfrak{S}(t) v$ is $L_{\text {loc }}^{1}\left(R^{n}\right)$-continuous for $t \geq 0$.

Combining Theorem I with the basic estimates for the operators $S_{t}$ as well as those for the approximate solutions to be shown later, we obtain the following result. (See §4.)

THEOREM II (PRODUCT FORMULA). There is a semigroup $\{\mathfrak{S}(t): t \geq 0\}$ of nonlinear operators on $L^{\infty}\left(R^{n}\right)$ with the following properties:

(i) For each $v \in L^{\infty}\left(R^{n}\right)$ the function $u(x, t)=[\mathfrak{S}(t) v](x)$ gives the entropy solution of $(\mathrm{M})$ with initial value $v$.

(ii) $\|\mathfrak{S}(t) v\|_{\infty} \leq e^{\alpha t}\left(\|v\|_{\infty}+t\|C\|_{\infty}\right)$ for $t \geq 0$ and $v \in L^{\infty}\left(R^{n}\right)$.

(iii) For each $v \in L^{\infty}\left(R^{n}\right)$ we have the product formula

$$
\mathfrak{S}(t) v=\lim _{h \rightarrow 0} S_{h}^{[t / h]} v \quad \text { in } L_{\text {loc }}^{1}\left(R^{n}\right)
$$

where the convergence holds uniformly for bounded $t$.

2. Estimates for approximate solutions. In this section we give basic estimates for approximate solutions $u^{h}$ and their derivatives. These estimates will be used in $\S 3$ to show the convergence of $u^{h}$. First we introduce weight functions $p_{r}(x), r>0$, which are used to obtain $L_{\text {loc }}^{1}$ estimates for $u^{h}$. Let $p$ be a smooth nonnegative function with compact support in $R^{n}$ such that $\int p(x) d x=1$ and set

$$
p_{r}(x)=\int p(x-y) \exp \left(-\delta_{r} \sum_{i=1}^{n}\left|y_{i}\right|\right) d y \text { for } x \in R^{n} \text { and } r>0,
$$

where the constant $\delta_{r}>0$ will be chosen in (2.3). The symbol $\|\cdot\|_{q}$ stands for the norm of $L^{q}\left(R^{n}\right), 1 \leq q \leq \infty$. We use the weighted norms $\|\cdot\|_{1, r}, r>0$, defined by

$$
\|v\|_{1, r}=\left\|p_{r} v\right\|_{1}, \quad v \in L^{\infty}\left(R^{n}\right)
$$

The following are easily verified:

(p.1) For $v \in L^{\infty}\left(R^{n}\right), p_{r} v \neq 0$ if $v \neq 0$.

(p.2) $\left\|p_{r} v\right\|_{\infty} \leq\|v\|_{\infty},\|v\|_{1, r} \leq\left\|p_{r}\right\|_{1}\|v\|_{\infty}=\left(2 / \delta_{r}\right)^{n}\|v\|_{\infty}$ for $v \in L^{\infty}\left(R^{n}\right)$.

(p.3) $p_{r}(x+y) \leq \exp \left(\delta_{r} \sum_{i=1}^{n}\left|y_{i}\right|\right) p_{r}(x)$.

We begin by establishing $L^{q}$ estimates $(q=1, \infty)$ for $S_{t} v$. In what follows we fix a number $\omega>0$ and set

$$
\delta_{r}=\frac{\omega}{M_{r}}, \quad M_{r}=\sum_{i=1}^{n} \sup \left\{\left|a^{i}(x, \xi)\right| ; x \in R^{n},|\xi| \leq r\right\} .
$$


LEMMA 2.1. Let $v$ and $w$ be in $L^{\infty}\left(R^{n}\right)$ and $C$ the function defined in (H.2). Choose $r>0$ so that $\|v\|_{\infty} \leq r,\|w\|_{\infty} \leq r$ and $\|C\|_{\infty} \leq r$. Then we have

$$
\left\|S_{t} v\right\|_{\infty} \leq e^{\alpha t}\left(\|v\|_{\infty}+t\|C\|_{\infty}\right) \leq r e^{\alpha t}(1+t)
$$

$$
\left\|S_{t} v\right\|_{1, r} \leq e^{(\beta+\omega) t}\|v\|_{1, r}+\int_{0}^{t} e^{(\beta+\omega)(t-\sigma)}\|C\|_{1, r} d \sigma
$$

for all $t \geq 0$.

Proof. Let $f_{0}(x, \xi)=F(v(x), \xi)$ for $(x, \xi) \in R^{n} \times R^{1}$. The function $f=$ $V_{\xi}(t) f_{0}$ is expressed as

$$
f(x, \xi, t)=f_{0}(y, \xi) \exp \left(-\int_{0}^{t} l(\sigma, \xi) d \sigma\right)+\int_{0}^{t} c(\sigma, \xi) \exp \left(-\int_{\sigma}^{t} l(\tau, \xi) d \tau\right) d \sigma
$$

where

$$
l(\sigma, \xi)=\sum_{i} a_{x_{i}}^{i}(z(\sigma), \xi)+b(z(\sigma), \xi),
$$

$c(\sigma, \xi)=c(z(\sigma), \xi)$, and $z(\sigma)=z(\sigma ; \xi)$ is a parametric representation of the characteristic curve associated with the linear problem $(\mathrm{m})$ such that $z(t)=x$ and $z(0)=y$. Let $\|v\|_{\infty}=r_{1}$ and $\|C\|_{\infty}=r_{2}$. From the definition of $F$ we have

$$
F\left(-r_{1}, \xi\right) \leq f_{0}(y, \xi) \leq f\left(r_{1}, \xi\right) \quad \text { and } \quad F\left(-r_{2}, \xi\right) \leq c(\sigma, \xi) \leq F\left(r_{2}, \xi\right)
$$

so that, by (H.3),

$$
\begin{aligned}
& F\left(-r_{1}, \xi\right) e^{\alpha t}+F\left(-r_{2}, \xi\right) \int_{0}^{t} e^{\alpha(t-\sigma)} d \sigma \\
& \quad \leq f(x, \xi, t) \leq F\left(r_{1}, \xi\right) e^{\alpha t}+F\left(r_{2}, \xi\right) \int_{0}^{t} e^{\alpha(t-\sigma)} d \sigma .
\end{aligned}
$$

This, together with condition (D), yields the estimate (i).

We next prove (ii). Let $f_{0}(x, \xi)=F(v(x), \xi)$ and $H_{t} v=\int U_{\xi}(t) f_{0} d \xi$. It suffices to show that

$$
\left\|H_{t} v\right\|_{1, r} \leq e^{(\beta+\omega) t}\|v\|_{1, r} \text { for all } t \geq 0 .
$$

Since $\left|U_{\xi}(t) f_{0}\right|=U_{\xi}(t)\left|f_{0}\right|$ by definition, we have

$$
\begin{aligned}
\left\|H_{t} v\right\|_{1, r} & \leq \int p_{r}(x) d x \int U_{\xi}(t)\left|f_{0}\right| d \xi=\int d \xi \int p_{r}(x) U_{\xi}(t)\left|f_{0}\right| d x \\
& =\int d \xi \int\left(U_{\xi}(t)^{*} p_{r}\right)(y)\left|f_{0}(y, \xi)\right| d y .
\end{aligned}
$$

Here $U_{\xi}(t)^{*}: L^{1}\left(R^{n}\right) \rightarrow L^{1}\left(R^{n}\right), t \geq 0$, denote the solution operators of the initial value problem

$$
g_{t}=\sum_{i=1}^{n} a^{i}(x, \xi) g_{x_{i}}-b(x, \xi) g,\left.\quad g\right|_{t=0}=g_{0} \in L^{1}\left(R^{n}\right) .
$$

Since $f_{0}$ vanishes for $|\xi|>r,($ H.3), (p.3) and (2.3) together imply that

$$
\begin{aligned}
\left(U_{\xi}(t)^{*} p_{r}\right)(y) & =p_{r}(x) \exp \left[\int_{t}^{0} b(z(\sigma), \xi) d \sigma\right] \leq e^{\beta t} p_{r}(y+(x-y)) \\
& \leq e^{\beta t} e^{\delta_{r} M_{r} t} p_{r}(y)=e^{(\beta+\omega) t} p_{r}(y) .
\end{aligned}
$$


This, together with (2.5), yields (2.4) since $|v|=\int|F(v, \xi)| d \xi$.

(iii) is proved in the same way as (ii) by using the identity

$$
|v-w|=\int|F(v, \xi)-F(w, \xi)| d \xi
$$

This completes the proof.

Applying Lemma 2.1 repeatedly, we obtain the following result.

Proposition 2.2. Let $u_{0}$ and $v_{0}$ be in $L^{\infty}\left(R^{n}\right)$. Let $u^{h}$ and $v^{h}$ denote the approximate solutions defined for initial values $u_{0}$ and $v_{0}$ by (1.1), respectively. Choose $r>0$ as in Lemma 2.1. If $T>0$ and $R \geq r e^{\alpha T}(1+T)$, then:

$$
\begin{gathered}
\left\|u^{h}(t)\right\|_{\infty} \leq e^{\alpha t}\left(\left\|u_{0}\right\|_{\infty}+t\|C\|_{\infty}\right) \leq R \\
\left\|u^{h}(t)\right\|_{1, R} \leq e^{(\beta+\omega) t}\left\|u_{0}\right\|_{1, R}+\int_{0}^{t} e^{(\beta+\omega)(t-\sigma)}\|C\|_{1, R} d \sigma \\
\left\|u^{h}(t)-v^{h}(t)\right\|_{1, R} \leq e^{(\beta+\omega) t}\left\|u_{0}-v_{0}\right\|_{1, R}
\end{gathered}
$$

for $t \in(0, T)$ and $h>0$, where $u^{h}(t)=u^{h}(\cdot, t)$ and $v^{h}(t)=v^{h}(\cdot, t)$.

We next give principal estimates for the derivatives $u_{x_{i}}^{h}, i=1, \ldots, n$. To this end we employ extended real-valued seminorms $\left\|D_{x} \cdot\right\|_{1, r}, r>0$, on $L^{\infty}\left(R^{n}\right)$ defined by

$$
\left\|D_{x} v\right\|_{1, r}=\sup \left\{\int \operatorname{div}\left(\psi p_{r}\right) v d x ; \psi \in\left(C_{0}^{1}\left(R^{n}\right)\right)^{n},|\psi| \leq 1 \text { on } R^{n}\right\} .
$$

The right-hand side is often denoted by $\int p_{r}\left|D_{x} v\right|$; see [6]. We then introduce a function space $\mathfrak{C}\left(R^{n}\right)$ which forms a core of the class of initial functions in question: $\mathfrak{C}\left(R^{n}\right)$ is defined as the set of those elements $v \in L^{\infty}\left(R^{n}\right)$ such that $\left\|D_{x} v\right\|_{1, r}$ is finite for all $r>0$. Notice that for $v \in \mathfrak{C}\left(R^{n}\right)$, the distributional derivatives $v_{x_{i}}$ are Radon measures with locally finite total variations. In what follows, $\left|D_{x} v\right|$ denotes the total variation measure of the vector-valued measure $D_{x} v=\left(v_{x_{1}}, \ldots, v_{x_{n}}\right)$, that is, the measure on the Borel $\sigma$-algebra of $R^{n}$ defined by the total variation of $D_{x} v$.

LEMMA 2.3. For each $v \in \mathfrak{C}\left(R^{n}\right)$ and each $r>0$, there exists a sequence $\left\{v_{m}\right\}$ of smooth functions on $R^{n}$ such that

$$
\left\|v_{m}-v\right\|_{1, r} \rightarrow 0, \quad\left\|D_{x} v_{m}\right\|_{1, r} \rightarrow\left\|D_{x} v\right\|_{1, r} \quad \text { as } m \rightarrow \infty .
$$

The proof of the above lemma is similar to those of [6, Theorem 1.17 and 12 , Kap. 6, Satz 1.1] so it is omitted.

LEMMA 2.4. For $v \in \mathfrak{C}\left(R^{n}\right)$ the derivatives $\partial F(v(\cdot), \xi) / \partial x_{i}$ are Radon measures for a.e. $\xi \in R^{1}$ and

$$
\left\|D_{x} v\right\|_{1, r}=\int\left\|D_{x} F(v(\cdot), \xi)\right\|_{1, r} d \xi \quad \text { for all } r>0
$$

ProOF. First we show that

$$
\int_{\Omega}\left|D_{x} v\right|=\int d \xi \int_{\Omega}\left|D_{x} F(v(\cdot), \xi)\right| \text { for any open set } \Omega
$$

where the left-hand side is defined by $(2.6)$ with $p_{r}$ replaced by the characteristic function $\chi_{\Omega}$ of $\Omega$ and $\psi \in\left(C_{0}^{1}(\Omega)\right)^{n}$. This relation is already known for the case 
$v \geq 0$; see [6, Theorem 1.23 or 12, Kap. 6, Satz 1.5]. For the general case we write $v=v^{+}-v^{-}$, where $v^{ \pm}=(|v| \pm v) / 2$, respectively. Then (see [12, p. 130])

$$
\begin{aligned}
\int_{\Omega}\left|D_{x} v\right| & =\int_{\Omega}\left|D_{x} v^{+}\right|+\int_{\Omega}\left|D_{x} v^{-}\right| \\
& =\int_{0}^{\infty} d \xi \int_{\Omega}\left|D_{x} F\left(v^{+}(\cdot), \xi\right)\right|+\int_{-\infty}^{0} d \xi \int_{\Omega}\left|D_{x} F\left(v^{-}(\cdot),-\xi\right)\right| .
\end{aligned}
$$

Since the identities $F(v, \xi)=F\left(v^{+}, \xi\right)-F\left(v^{-}, \xi\right), F\left(v^{ \pm}, \pm \xi\right)=F(v, \xi)^{ \pm}$hold pointwise for $x \in R^{n}$, the right-hand side equals

$$
\int d \xi \int_{\Omega}\left|D_{x} F(v(\cdot), \xi)^{+}\right|+\int d \xi \int_{\Omega}\left|D_{x} F(v(\cdot), \xi)^{-}\right|=\int d \xi \int_{\Omega}\left|D_{x} F(v(\cdot), \xi)\right| .
$$

This proves (2.7). The regularity property of the Radon measure now implies that (2.7) holds for any Borel set $\Omega$. Thus, approximating $p_{r}$ by simple functions, we obtain the desired result.

LEMMA 2.5. Let $\|C\|_{\infty} \leq r$ and choose $\gamma \geq 0$ so that

$$
\gamma \geq\left\{\begin{array}{l}
\sum_{i, j} \sup \left\{\left|a_{x_{j}}^{i}(x, \xi)\right| ; x \in R^{n},|\xi| \leq r\right\}, \\
\sum_{i, j} \sup \left\{\left|a_{x_{i} x_{j}}^{i}(x, \xi)+b_{x_{j}}(x, \xi)\right| ; x \in R^{n},|\xi| \leq r\right\} .
\end{array}\right.
$$

If $v \in \mathfrak{C}\left(R^{n}\right)$ and $\|v\|_{\infty} \leq r$, we have

$$
\begin{aligned}
\left\|D_{x} S_{t} v\right\|_{1, r} \leq & e^{(\beta+\omega+\gamma) t}\left(\left\|D_{x} v\right\|_{1, r}+\gamma t\|v\|_{1, r}\right) \\
& +\int_{0}^{t} e^{(\beta+\omega+\gamma)(t-\sigma)}\left(\left\|D_{x} C\right\|_{1, r}+\gamma(t-\sigma)\|C\|_{1, r}\right) d \sigma
\end{aligned}
$$

for all $t \geq 0$.

Proof. Let $f_{0}(x, \xi)=F(v(x), \xi)$ and $f=V_{\xi}(t) f_{0}$. We wish to show that

$$
\begin{aligned}
& \left\|D_{x} f(\cdot, \xi, t)\right\|_{1, r} \leq e^{(\beta+\omega+\gamma) t}\left(\left\|D_{x} f_{0}(\cdot, \xi)\right\|_{1, r}+\gamma t\left\|f_{0}(\cdot, \xi)\right\|_{1, r}\right) \\
& \quad+\int_{0}^{t} e^{(\beta+\omega+\gamma)(t-\sigma)}\left[\left\|D_{x} c(\cdot, \xi)\right\|_{1, r}+\gamma(t-\sigma)\|c(\cdot, \xi)\|_{1, r}\right] d \sigma
\end{aligned}
$$

holds for $t \geq 0$ and $|\xi| \leq r$. The desired estimate is then obtained by integrating (2.8) with respect to $\xi$ and applying Lemma 2.4 to the resulting inequality. By Lemma 2.3 we may assume that $f_{0}$ and $c$ are smooth in $x$ for each $\xi$ with $|\xi| \leq r$. In this case $f_{j} \equiv f_{x_{j}}$ satisfies the equation

$$
\left(f_{j}\right)_{t}+\sum_{i=1}^{n}\left(a^{i} f_{j}\right)_{x_{i}}+b f_{j}=c_{x_{i}}-b_{x_{j}} f-\sum_{i=1}^{n} a_{x_{i} x_{j}}^{i} f-\sum_{i=1}^{n} a_{x_{j}}^{i} f_{i}
$$

so that, as in the proof of Lemma 2.1(ii), we have

$$
\begin{aligned}
\left\|D_{x} f(\cdot, \xi, t)\right\|_{1, r} \leq & e^{(\beta+\omega) t}\left\|D_{x} f_{0}(\cdot, \xi)\right\|_{1, r} \\
& +\int_{0}^{t} e^{(\beta+\omega)(t-\sigma)}\left[\left\|D_{x} c(\cdot, \xi)\right\|_{1, r}+\gamma\|f(\cdot, \xi, \sigma)\|_{1, r}\right] d \sigma \\
& +\gamma \int_{0}^{t} e^{(\beta+\omega)(t-\sigma)}\left\|D_{x} f(\cdot, \xi, \sigma)\right\|_{1, r} d \sigma
\end{aligned}
$$


and

$$
\|f(\cdot, \xi, \sigma)\|_{1, r} \leq e^{(\beta+\omega) \sigma}\left\|f_{0}(\cdot, \xi)\right\|_{1, r}+\int_{0}^{\sigma} e^{(\beta+\omega)(\sigma-\tau)}\|c(\cdot, \xi)\|_{1, r} d \tau
$$

These estimates together yield

$$
\begin{aligned}
\left\|D_{x} f(\cdot, \xi, t)\right\|_{1, r} \leq & e^{(\beta+\omega) t}\left[\left\|D_{x} f_{0}(\cdot, \xi)\right\|_{1, r}+\gamma t\left\|f_{0}(\cdot, \xi)\right\|_{1, r}\right] \\
& +\int_{0}^{t} e^{(\beta+\omega)(t-\sigma)}\left[\left\|D_{x} c(\cdot, \xi)\right\|_{1, r}+\gamma(t-\sigma)\|c(\cdot, \xi)\|_{1, r}\right] d \sigma \\
& +\gamma \int_{0}^{t} e^{(\beta+\omega)(t-\sigma)}\left\|D_{x} f(\cdot, \xi, \sigma)\right\|_{1, r} d \sigma .
\end{aligned}
$$

Applying Gronwall's lemma to $G(\sigma)=\left\|D_{x} f(\cdot, \xi, \sigma)\right\|_{1, r}$, we obtain (2.8).

Using Lemma 2.5 repeatedly, we obtain

PROPOSITION 2.6. Given $T>0$, let $R \geq r e^{\alpha T}(1+T)$. Define $\gamma$ as in Lemma 2.5 with $r$ replaced by $R$. Let $u_{0} \in \mathfrak{C}\left(R^{n}\right)$ and $\left\|u_{0}\right\|_{\infty} \leq r$. Then

$$
\begin{aligned}
\left\|D_{x} u^{h}(\cdot, t)\right\|_{1, R} \leq & e^{(\beta+\omega+\gamma) t}\left(\left\|D_{x} u_{0}\right\|_{1, R}+\gamma t\left\|u_{0}\right\|_{1, r}\right) \\
& +e \int_{0}^{t} e^{(\beta+\omega+\gamma)(t-\sigma)}\left[\left\|D_{x} C\right\|_{1, R}+\gamma(t-\sigma)\|C\|_{1, R}\right] d \sigma
\end{aligned}
$$

for $t \in(0, T)$ and $h>0$, where $C$ is the function defined in (H.2).

We now compute the variations of the derivatives $\left(S_{t} v\right)_{t}$ and $\left(u^{h}\right)_{t}$ by using Lemma 2.5 and Proposition 2.6. Put

$$
\begin{aligned}
z(x, t) & =\int b(x, \xi) V_{\xi}(t) f_{0} d \xi \\
y^{i}(x, t) & =\int a^{i}(x, \xi) V_{\xi}(t) f_{0} d \xi, \quad i=1, \ldots, n,
\end{aligned}
$$

where $f_{0}=F(v, \xi)$ and $v \in L^{\infty}\left(R^{n}\right)$. Then it is clear that the relation

$$
\left(S_{t} v\right)_{t}+\sum_{i=1}^{n} y_{x_{i}}^{i}+z=C
$$

holds on $R^{n} \times(0, \infty)$ in the sense of distributions. Using this, we obtain the following.

LEMMA 2.7. For each $v \in \mathfrak{C}\left(R^{n}\right)$ with $\|v\|_{\infty} \leq r$ and each $t \geq 0$, the derivative $\left(S_{t} v\right)_{t}(\cdot, t)$ is a Radon measure on $R^{n}$ and is estimated as

$$
\begin{aligned}
&\left\|\left(S_{t} v\right)_{t}(\cdot, t)\right\|_{1, r} \leq\|C\|_{1, r}+K_{r}\left[e^{(\beta+\omega) t}\|v\|_{1, r}+\int_{0}^{t} e^{(\beta+\omega)(t-\sigma)}\|C\|_{1, r} d \sigma\right] \\
&+M_{r}\left[e^{(\beta+\omega+\gamma) t}\left(\left\|D_{x} v\right\|_{1, r}+\gamma t\|v\|_{1, r}\right)\right. \\
&\left.\quad+\int_{0}^{t} e^{(\beta+\omega+\gamma)(t-\sigma)}\left(\left\|D_{x} C\right\|_{1, r}+\gamma(t-\sigma)\|C\|_{1, r}\right) d \sigma\right] .
\end{aligned}
$$

Here $M_{r}$ is the number defined in (2.3),

$$
K_{r}=\sup \left\{|b(x, \xi)|+\sum_{i}\left|a_{x_{i}}^{i}(x, \xi)\right| ; x \in R^{n},|\xi| \leq r\right\}
$$


and $\left\|\left(S_{t} v\right)_{t}(\cdot, t)\right\|_{1, r}$ denotes the integral of $p_{r}$ with respect to the total variation of the measure $\left(S_{t} v\right)_{t}(\cdot, t)$. In particular, for each $T>0$, there is a constant $K>0$ such that

$$
\left\|S_{t} v-S_{\tau} v\right\|_{1, r} \leq K|t-\tau| \text { for } t, \tau \in[0, T] .
$$

The next result is an immediate consequence of Lemma 2.7.

Proposition 2.8. Let $T>0$ and $R \geq r e^{\alpha T}(1+T)$. Define $\gamma$ as in Lemma 2.5 with $r$ replaced by $R$. If $u_{0} \in \mathfrak{C}\left(R^{n}\right)$ and $\left\|u_{0}\right\|_{\infty} \leq r$, then there is a constant $K>0$ depending only on $R, \gamma$ and $\left\|D_{x} u_{0}\right\|_{1, r}$ such that

$$
\left\|u^{h}(t)-u^{h}(s)\right\|_{1, R} \leq K|t-s|
$$

for $t, s \in[0, T]$ and $h>0$.

3. Convergence to the entropy solutions. Propositions $2.2,2.6$ and 2.8 show that the weighted norms of $u^{h}$ are uniformly bounded on $[0, T]$, and the total variations of $u^{h}$ are uniformly bounded on each compact subset of $R^{n} \times(0, T)$. Further, estimate (2.9) implies

$$
\left\|u^{h}(t)-S_{h}^{[t / h]} u_{0}\right\|_{1, R} \leq K(t-h[t / h]) \quad \text { for } t \in[0, T], h>0 .
$$

Thus, a well-known compactness theorem ([6, Theorem 1.19]) yields the following.

Proposition 3.1. Let $u_{0}$ be in $\mathfrak{C}\left(R^{n}\right)$. Then there exist a sequence $h(m) \rightarrow 0$ and a function $u$ on $R^{n} \times(0, \infty)$ with the following properties:

(i) $u^{h(m)}(\cdot, t) \rightarrow u(\cdot, t), S_{h(m)}^{[t / h(m)]} u_{0} \rightarrow u(\cdot, t)$ in $L_{\text {loc }}^{1}\left(R^{n}\right)$ where the convergence is uniform for $t$ in bounded subintervals of $[0, \infty)$.

(ii) $u$ is in $L^{\infty}\left(R^{n} \times(0, T)\right)$ for every $T>0$.

(iii) The mapt $\rightarrow u(\cdot, t)$ is continuous from $[0, \infty)$ into $L_{\text {loc }}^{1}\left(R^{n}\right)$ and $u(\cdot, 0)=u_{0}$.

Notice that the uniformity in $t \geq 0$ of the convergence (i) follows from (2.9). In this section we first show that the function $u$ given above is the entropy solution of (M). Since the entropy solution is known to be unique, it turns out that $\left\{u^{h}\right\}$ itself tends to $u$ as $h \rightarrow 0$, and Theorem I (Convergence Theorem) is obtained for $u_{0} \in \mathfrak{C}\left(R^{n}\right)$. This result will then be applied to show the convergence of $\left\{u^{h}\right\}$ for an arbitrary $u_{0}$ in $L^{\infty}\left(R^{n}\right)$. In what follows, we set

$$
\mathfrak{A}_{h}=h^{-1}\left(S_{h}-1\right), \quad h>0 .
$$

To prove Theorem I for $u_{0} \in \mathfrak{C}\left(R^{n}\right)$, we need a few lemmas.

LEMMA 3.2. Let $u_{0} \in \mathfrak{C}\left(R^{n}\right)$. Then, for each $h \in(0,1)$, there is a number $\lambda_{0}=\lambda_{0}(h)>0$ such that:

(i) $u_{h}^{\lambda}(t) \equiv\left(1-\lambda \mathfrak{A}_{h}\right)^{-[t / \lambda]} u_{0}$ is meaningful for $\lambda \in\left(0, \lambda_{0}\right)$ and $t \geq 0$;

(ii) there is a function $u_{h}(t)$ such that $u_{n}^{\lambda}(t) \rightarrow u_{h}(t)$ in $L_{\mathrm{loc}}^{1}\left(R^{n}\right)$ as $\lambda \rightarrow 0$, uniformly for bounded $t$.

LEMma 3.3. Let $u_{0}$ and $u_{h}(t)$ be as in Lemma 3.2. Then

$$
S_{h}^{[t / h]} u_{0}-u_{h}(t) \rightarrow 0 \text { in } L_{\text {loc }}^{1}\left(R^{n}\right) \quad \text { as } h \rightarrow 0
$$

uniformly for bounded $t$.

Lemma 3.2 is a modified version to the case of the Fréchet space $L_{\text {loc }}^{1}\left(R^{n}\right)$ of the generation theorem of nonlinear semigroups due to Crandall and Liggett $[\mathbf{2}]$; and 
Lemma 3.3 is similar to an approximation theorem for nonlinear semigroups due to Brezis and Pazy [1] and Miyadera and Kobayshi [13]. The proofs of these lemmas is given in $\S 4$.

The next lemma may be regarded as a discrete version of inequality (E).

LEMMA 3.4. For $v \in L^{\infty}\left(R^{n}\right), s \in R^{1}$ and $\psi \in C_{0}^{\infty}\left(R^{n}\right)$ with $\psi \geq 0$, we have

$$
\begin{aligned}
\int \operatorname{sgn}(v-s) & \psi \mathfrak{A}_{h} v d x \\
\leq & \iint \operatorname{sgn}(v-s)(F(v, \xi)-F(s, \xi))\left[h^{-1}\left(U_{\xi}(h)^{*}-1\right) \psi\right] d x d \xi \\
& +\iint \operatorname{sgn}(v-s) \psi\left[h^{-1}\left(U_{\xi}(h)-1\right) F(s, \xi)\right] d x d \xi \\
& +h^{-1} \iint \operatorname{sgn}(v-s) \psi\left[\int_{0}^{h} U_{\xi}(h-\sigma) c(\cdot, \xi) d \sigma\right] d x d \xi
\end{aligned}
$$

where $U_{\xi}(t)^{*}$ are the solution operators of problem $(\mathrm{m})^{*}$.

ProOF. By definition of $\mathfrak{A}_{h}$,

$$
\begin{aligned}
\int \operatorname{sgn}(v-s) \psi \mathfrak{A}_{h} v d x= & \iint \operatorname{sgn}(v-s) \psi\left[h^{-1}\left(U_{\xi}(h)-1\right)(F(v, \xi)-F(s, \xi))\right] d x d \xi \\
& +\iint \operatorname{sgn}(v-s) \psi\left[h^{-1}\left(U_{\xi}(h)-1\right) F(s, \xi)\right] d x d \xi \\
& +h^{-1} \iint \operatorname{sgn}(v-s) \psi\left[\int_{0}^{h} U_{\xi}(h-\sigma) c(\cdot, \xi) d \sigma\right] d x d \xi .
\end{aligned}
$$

The first term on the right-hand side is transformed to

$$
\begin{aligned}
\iint(F(v, \xi)-F(s, \xi)) h^{-1}\left(U_{\xi}(h)^{*}-1\right)(\operatorname{sgn}(v-s) \psi) d x d \xi \\
=\iint(F(v, \xi)-F(s, \xi)) \operatorname{sgn}(v-s) h^{-1}\left(U_{\xi}(h)^{*}-1\right) d x d \xi \\
+h^{-1} \iint(F(v, \xi)-F(s, \xi)) \\
\times\left[U_{\xi}(h)^{*}(\operatorname{sgn}(v-s) \psi)-\operatorname{sgn}(v-s) U_{\xi}(h)^{*} \psi\right] d x d \xi
\end{aligned}
$$

Since $(F(v, \xi)-F(s, \xi)) \operatorname{sgn}(v-s)=|F(v, \xi)-F(s, \xi)|$ and $U_{\xi}(h)^{*}$ is orderpreserving, we easily see that

$$
(F(v, \xi)-F(s, \xi))\left[U_{\xi}(h)^{*}(\operatorname{sgn}(v-s) \psi)-\operatorname{sgn}(v-s) U_{\xi}(h)^{*} \psi\right] \leq 0
$$

for a.e. $(x, \xi) \in R^{n+1}$. This shows the desired estimate (3.2).

Let $u$ be the function given in Proposition 3.1. We wish to prove that $u$ satisfies inequality (E). Hereafter we denote $h(m)$ by $h$ for simplicity in notation. Choose any $\phi \in C_{0}^{\infty}\left(R^{n} \times(0, \infty)\right)$ with $\phi \geq 0$, and let $v(x)=u_{h}^{\lambda}(x, t), \psi(x)=\phi(x, t)$ in (3.2). Since

$$
\left(\mathfrak{A}_{h} u_{h}^{\lambda}\right)(x, t)=\lambda^{-1}\left(u_{h}^{\lambda}(x, t)-u_{h}^{\lambda}(x, t-\lambda)\right),
$$

we have

$$
\operatorname{sgn}\left(u_{h}^{\lambda}(x, t)-s\right)\left(\mathfrak{A}_{h} u_{h}^{\lambda}\right)(x, t) \geq \lambda^{-1}\left(\left|u_{h}^{\lambda}(x, t)-s\right|-\left|u_{h}^{\lambda}(x, t-\lambda)-s\right|\right),
$$


and so

$$
\begin{aligned}
\int_{\lambda}^{\infty} & d t \int \phi(x, t)\left(\mathfrak{A}_{h} u_{h}^{\lambda}\right)(x, t) \operatorname{sgn}\left(u_{h}^{\lambda}(x, t)-s\right) d x \\
& \geq \lambda^{-1} \int_{0}^{\infty} d t \int(\phi(x, t)-\phi(x, t+\lambda))\left|u_{h}^{\lambda}(x, t)-s\right| d x
\end{aligned}
$$

for small $\lambda$, since $\phi \in C_{0}^{\infty}\left(R^{n} \times(0, \infty)\right)$. On the other hand, (3.2) gives

$$
\begin{aligned}
\int_{\lambda}^{\infty} d t & \int \phi(x, t)\left(\mathfrak{A}_{h} u_{h}^{\lambda}\right)(x, t) \operatorname{sgn}\left(u_{h}^{\lambda}(x, t)-s\right) d x \\
\leq & \int_{\lambda}^{\infty} d t \iint \operatorname{sgn}\left(u_{h}^{\lambda}-s\right)\left(F\left(u_{h}^{\lambda}, s\right)-F(s, \xi)\right)\left[h^{-1} \int_{0}^{h} U_{\xi}(\sigma)^{*} L^{*} \phi d \sigma\right] d x d \xi \\
& +\int_{\lambda}^{\infty} d t \iint \operatorname{sgn}\left(u_{h}^{\lambda}-s\right)\left[h^{-1} \int_{0}^{h} U_{\xi}(\sigma) L F(s, \xi) d \sigma\right] d x d \xi \\
& +\int_{\lambda}^{\infty} d t \iint \operatorname{sgn}\left(u_{h}^{\lambda}-s\right) \phi\left[h^{-1} \int_{0}^{h} U_{\xi}(h-\sigma) c(\cdot, \xi) d \sigma\right] d x d \xi \\
\equiv & I_{1}(s, h, \lambda)+I_{2}(s, h, \lambda)+I_{3}(s, h, \lambda),
\end{aligned}
$$

where

$$
L g=-\sum_{i}\left(a^{i} g\right)_{x_{i}}-b g, \quad L^{*} g=\sum_{i} a^{i} g_{x_{i}}-b g
$$

Let $\Phi_{j}(s), j=1,2, \ldots$, be functions on $R^{1}$ defined by

$$
\Phi_{j}(s)= \begin{cases}|s| & \text { for }|s| \geq 1 / j \\ j s^{2} / 2+1 / 2 j & \text { for }|s| \leq 1 / j\end{cases}
$$

We easily see that

$$
\lim _{j \rightarrow \infty} \int_{k}^{y} \Phi_{j}^{\prime \prime}(s-k) g(s) d s=\operatorname{sgn}(y-k) g(k) \text { for } g \in C^{1}\left(R^{1}\right) .
$$

Now fix $k \in R^{1}$ and set $q_{j}(s)=\Phi_{j}^{\prime \prime}(s-k)$. Since $q_{j} \geq 0,(3.4)$ gives

$$
\int_{-\infty}^{\infty} q_{j}(s) d s \int_{\lambda}^{\infty} d t \int \operatorname{sgn}\left(u_{h}^{\lambda}-s\right) \phi A_{h} u_{h}^{\lambda} d x \leq \sum_{m=1}^{3} \int_{-\infty}^{\infty} q_{j}(s) I_{m}(s, h, \lambda) d s
$$

We write $J_{m} \equiv J_{m}(h, \lambda, j)$ for the integrals on the right side of (3.7) and wish to find the limit of each $J_{m}$ as $\lambda \rightarrow 0, h \rightarrow 0$ and then $j \rightarrow \infty$. We only give a detailed argument to $J_{1}$ since $J_{m}, m=2,3$, are similarly treated. First $J_{1}$ is written as

$$
\begin{aligned}
J_{1}= & \int_{-\infty}^{\infty} q_{j}(s) d s \int_{\lambda}^{\infty} d t \iint \operatorname{sgn}\left(u_{h}^{\lambda}-s\right)\left(F\left(u_{h}^{\lambda}, \xi\right)-F(s, \xi)\right) L^{*} \phi d x d \xi \\
+ & \int_{-\infty}^{\infty} q_{j}(s) d s \int_{\lambda}^{\infty} d t \iint \operatorname{sgn}\left(u_{h}^{\lambda}-s\right)\left(F\left(u_{h}^{\lambda}, \xi\right)-F(s, \xi)\right) \\
& \cdot\left[h^{-1} \int_{0}^{h}\left(U_{\xi}(\sigma)^{*}-1\right) L^{*} \phi d \sigma\right] d x d \xi \\
\equiv & J_{11}+J_{12} .
\end{aligned}
$$


Condition (C), (3.5) and the definition of $q_{j}$ yield

$$
\begin{aligned}
J_{11}= & \int_{-\infty}^{\infty} q_{j}(s) d s \int_{\lambda}^{\infty} d t \int \operatorname{sgn}\left(u_{h}^{\lambda}-s\right)\left(A\left(x, u_{h}^{\lambda}\right)-A(x, s)\right) \cdot \nabla_{x} \phi d x \\
& -\int_{-\infty}^{\infty} q_{j}(s) d s \int_{\lambda}^{\infty} d t \int \operatorname{sgn}\left(u_{h}^{\lambda}-s\right)\left(B\left(x, u_{h}^{\lambda}\right)-B(x, s)\right) \phi d x \\
= & \int_{\lambda}^{\infty} d t \int d x\left[\int_{-\infty}^{k}-\int_{k}^{\infty}+2 \int_{k}^{u_{h}^{\lambda}}\right] q_{j}(s)\left(A\left(x, u_{h}^{\lambda}\right)-A(x, s)\right) \cdot \nabla_{x} \phi d x \\
& -\int_{\lambda}^{\infty} d t \int d x\left[\int_{-\infty}^{k}-\int_{k}^{\infty}+2 \int_{k}^{u_{h}^{\lambda}}\right] q_{j}(s)\left(B\left(x, u_{h}^{\lambda}\right)-B(x, s)\right) \phi d s
\end{aligned}
$$

where

$$
(A(x, w)-A(x, s)) \cdot \nabla_{x} \phi=\sum_{i}\left(A^{i}(x, w)-A^{i}(x, s)\right) \phi_{x_{i}} .
$$

As will be seen in $\S 4,\left\{u_{h}^{\lambda}\right\}$ is bounded in $L^{\infty}\left(R^{n} \times(0, T)\right)$ for any $T>0$. Also, by Proposition 3.1, Lemmas 3.2 and 3.3, we may assume without loss of generality that $\lim _{h \rightarrow 0} \lim _{\lambda \rightarrow 0} u_{h}^{\lambda}=u$ a.e. in $R^{n} \times(0, \infty)$. Hence (3.6) implies that

$$
\begin{aligned}
\lim _{j \rightarrow \infty} \lim _{h \rightarrow 0} \lim _{\lambda \rightarrow 0} J_{11}(h, \lambda, j) \\
=2 \int_{0}^{\infty} d t \int \operatorname{sgn}(u-k)(A(x, u)-A(x, k)) \cdot \nabla_{x} \phi d x \\
-2 \int_{0}^{\infty} d t \int \operatorname{sgn}(u-k)(B(x, u)-B(x, k)) \phi d x .
\end{aligned}
$$

We next consider $J_{12}$. The function $g=U_{\xi}(\sigma)^{*} L^{*} \phi$ solves the initial value problem $g_{\sigma}=L^{*} g(\sigma>0),\left.g\right|_{\sigma=0}=L^{*} \phi$. So we see from the same arguments as in the proofs of Lemma 2.7 and (2.8) that for each $\gamma>0$ a constant $K_{\gamma}$ can be found so that

$$
\int_{E}\left|\left(U_{\xi}(\sigma)^{*}-1\right) L^{*} \phi\right| d x \leq K_{\gamma} \sigma \quad \text { for } \sigma \in[0, h] \text { and }|\xi| \leq \gamma,
$$

where $E=\left\{x \in R^{n} ;(x, t) \in \operatorname{supp} \phi\right.$ for some $\left.t>0\right\}$. Since $\left\{u_{h}^{\lambda}\right\}$ is bounded in $L^{\infty}\left(R^{n} \times(0, T)\right)$ for any $T>0$ and since $\operatorname{supp} q_{j}=[k-1 / j, k+1 / j],(3.9)$ implies that

$$
\left|J_{12}\right| \leq \text { const } \times\left(\int_{-\infty}^{\infty} q_{j}(s) d s\right) \times h \rightarrow 0
$$

as $\lambda \rightarrow 0, h \rightarrow 0$ and then $j \rightarrow \infty$. (Notice that $\int_{-\infty}^{\infty} q_{j}(s) d s=2$.) Similarly, one can show that

$$
\begin{aligned}
& \lim _{j \rightarrow \infty} \lim _{h \rightarrow 0} \lim _{\lambda \rightarrow 0} J_{2}(h, \lambda, j) \\
& \quad=-2 \int_{0}^{\infty} d t \int \operatorname{sgn}(u-k)\left[\sum_{i} A_{x_{i}}^{i}(x, k)+B(x, k)+C(x)\right] \phi d x
\end{aligned}
$$

and

$$
\lim _{j \rightarrow \infty} \lim _{h \rightarrow 0} \lim _{\lambda \rightarrow 0} J_{3}(h, \lambda, j)=2 \int_{0}^{\infty} d t \int \operatorname{sgn}(u-k) C(x) \phi d x
$$


where $C(x)$ is the function defined in (H.2). From (3.8) and (3.10)-(3.12) we have (3.13)

$$
\begin{aligned}
\lim _{j \rightarrow \infty} \lim _{h \rightarrow 0} \lim _{\lambda \rightarrow 0} \sum_{m=1}^{3} J_{m}= & 2 \int_{0}^{\infty} d t \int \operatorname{sgn}(u-k)(A(x, u)-A(x, k)) \cdot \nabla_{x} \phi d x \\
& -2 \int_{0}^{\infty} d t \int \operatorname{sgn}(u-k)\left[\sum_{i} A_{x_{i}}^{i}(x, k)+B(x, u)\right] \phi d x .
\end{aligned}
$$

On the other hand, it follows from (3.3) and (3.7) that

$$
\begin{aligned}
\sum_{m=1}^{3} J_{m} & \geq \frac{1}{\lambda} \int_{-\infty}^{\infty} q_{j}(s) d s \int_{0}^{\infty} d t \int(\phi(x, t)-\phi(x, t+\lambda))\left|u_{h}^{\lambda}(x, t)-s\right| d x \\
& \rightarrow-2 \int_{0}^{\infty} d t \int \phi_{t}|u-k| d x
\end{aligned}
$$

as $\lambda \rightarrow 0, h \rightarrow 0$ and then $j \rightarrow \infty$. (3.13) and (3.14) show that $u$ satisfies inequality (E), which completes the proof of the Convergence Theorem for the case $u_{0} \in \mathfrak{C}\left(R^{n}\right)$.

We now consider the general case: $u_{0} \in L^{\infty}\left(R^{n}\right)$. Fix any $T>0$ and choose $r>0$ so that $\left\|u_{0}\right\|_{\infty} \leq r$ and $\|C\|_{\infty} \leq r$. Let $R=r e^{\alpha T}(1+T)$ and let $\left\{u_{0 m}\right\}$ be a sequence of functions in $\mathfrak{C}\left(R^{n}\right)$ such that $\left\|u_{0 m}\right\|_{\infty} \leq r$ and

$$
\left\|u_{0 m}-u_{0}\right\|_{1, R} \rightarrow 0 \text { as } m \rightarrow \infty
$$

where $\|\cdot\|_{1, R}$ is the weighted norm defined by (2.2). Let

$$
u_{m}^{h}(x, t)=\left(S_{t-h[t / h]} S_{h}^{[t / h]} u_{0 m}\right)(x)
$$

and $u_{m}$ be the entropy solution of $(\mathrm{M})$ with $u_{m}(0)=u_{0 m}$. By Proposition 2.2,

$$
\left\|u_{l}^{h}(t)-u_{m}^{h}(t)\right\|_{1, R} \leq e^{(\beta+\omega) t}\left\|u_{0 l}-u_{0 m}\right\|_{1, R} \quad \text { for } t \in(0, T) .
$$

Letting $h \rightarrow 0$, we obtain

$$
\left\|u_{l}(t)-u_{m}(t)\right\|_{1, R} \leq e^{(\beta+\omega) t}\left\|u_{0 l}-u_{0 m}\right\|_{1, R} \quad \text { for } t \in(0, T) .
$$

Hence (3.15) implies that $u_{m}(t)$ converges in $L_{\text {loc }}^{1}\left(R^{n}\right)$ to a function $u(t)$ uniformly on $[0, T]$ in such a way that

$$
\left\|u(t)-u_{m}(t)\right\|_{1, R} \leq e^{(\beta+\omega) t}\left\|u_{0}-u_{0 m}\right\|_{1, R} \quad \text { for } t \in(0, T) .
$$

We shall show that $u(t)$ is the desired entropy solution. Since $u_{m}$ are continuous from $[0, T]$ into $L_{\text {loc }}^{1}\left(R^{n}\right)$ by Proposition 3.1, so is $u$. Thus, $u(t) \rightarrow u_{0}$ in $L_{\text {loc }}^{1}\left(R^{n}\right)$ as $t \rightarrow 0$. Further, Proposition 2.2(i) implies that $\left\|u_{m}^{h}(t)\right\| \leq R$ for $t \in(0, T)$, so $u \in L^{\infty}\left(R^{n} \times(0, T)\right)$. Next we show that, as $h \rightarrow 0$,

$$
u^{h}(t) \rightarrow u(t) \text { in } L_{\text {loc }}^{1}\left(R^{n}\right) \text { uniformly on }[0, T]
$$

Indeed, Proposition 2.2(iii) and (3.17) imply

$$
\begin{aligned}
\left\|u^{h}(t)-u(t)\right\|_{1, R} & \\
& \leq\left\|u^{h}(t)-u_{m}^{h}(t)\right\|_{1, R}+\left\|u_{m}^{h}(t)-u_{m}(t)\right\|_{1, R}+\left\|u_{m}(t)-u(t)\right\|_{1, R} \\
& \leq\left\|u_{m}^{h}(t)-u_{m}(t)\right\|_{1, R}+2 e^{(\beta+\omega) t}\left\|u_{0 m}-u_{0}\right\|_{1, R}
\end{aligned}
$$


for all $t \in(0, T)$. Since $p_{R}$ is integrable and bounded on $R^{n}$, the estimates $\left\|u_{m}^{h}(t)\right\|_{\infty} \leq R,\left\|u_{m}(t)\right\|_{\infty} \leq R$ and Proposition 3.1(i) imply that

$$
\left\|u_{m}^{h}(t)-u_{m}(t)\right\|_{1, R} \rightarrow 0 \quad \text { as } h \rightarrow 0 \text { uniformly on }[0, T]
$$

Hence (3.18) follows from (3.15). On the other hand, each $u_{m}$ satisfies inequality (E), so

$$
\begin{aligned}
\int_{-\infty}^{\infty} q_{j}(s) d s & {\left[\int_{0}^{\infty} \int \phi_{t}\left|u_{m}-s\right| d x d t\right.} \\
+ & \left.\int_{0}^{\infty} \int \operatorname{sgn}\left(u_{m}-s\right)\left(A\left(x, u_{m}\right)-A(x, s)\right) \cdot \nabla_{x} \phi d x d t\right] \\
& -\int_{-\infty}^{\infty} q_{j}(s) d s \int_{0}^{\infty} \int \operatorname{sgn}\left(u_{m}-s\right)\left[\sum_{i} A_{x_{i}}^{i}(x, s)+B\left(x, u_{m}\right)\right] \phi d x \geq 0
\end{aligned}
$$

for every nonnegative $\phi \in C_{0}^{\infty}\left(R^{n} \times(0, \infty)\right)$. Passing to the limit as in the case $u_{0} \in \mathfrak{C}\left(R^{n}\right)$, we see that $u$ satisfies inequality (E); therefore the proof is complete.

4. A nonlinear semigroup in $L_{\text {loc }}^{1}\left(R^{n}\right)$. In this section we establish a product formula for a nonlinear semigroup associated with problem (M) in $L_{\text {loc }}^{1}\left(R^{n}\right)$, and give a result for this semigroup in a more precise form than Theorem II. This section is divided into three subsections. $\S 4.1$ contains the proof of Lemma 3.2, $\S 4.2$ is devoted to the verification of Lema 3.3 , and in $\S 4.3$, a nonlinear semigroup on $L^{\infty}\left(R^{n}\right)$, as mentioned in (1.3)-(1.5), is constructed through the product formula (1.6).

4.1. Proof of Lemma 3.2. We begin by defining the numbers

$$
\begin{gathered}
\alpha_{0}=\alpha_{0}(h)=h^{-1}\left(e^{\alpha h}-1\right), \quad \beta_{0}=\beta_{0}(h)=h^{-1}\left(e^{(\beta+\omega) h}-1\right), \\
\lambda_{0}=\lambda_{0}(h)=\min \left\{1 / 2 \alpha_{0}(h), 1 / \beta_{0}(h)\right\}
\end{gathered}
$$

for each $h>0$, where $\alpha, \beta$ and $\omega$ are constants specified in (H.3) and (2.3). Further, we often use the operators $\mathfrak{A}_{h}, h>0$, defined by (3.1), in the argument below:

$$
\mathfrak{A}_{h}=h^{-1}\left(S_{h}-1\right), \quad h>0 .
$$

First we need the following lemma.

LEMMA 4.1. Let $h \in(0,1)$ and let $r>0$ be any number such that $\|C\|_{\infty} \leq r$, where $C$ is the function defined in condition (H.2). Let $\lambda \in\left(0, \lambda_{0}\right)$ and $r^{\prime} \geq$ $\left(1-\lambda \alpha_{0}\right)^{-1}\left(1+\lambda e^{\alpha h}\right) r$. Then, for each $w \in L^{\infty}\left(R^{n}\right)$ with $\|w\|_{\infty} \leq r$ there is a unique function $v \in L^{\infty}\left(R^{n}\right)$ such that

(i) $\left(1-\lambda \mathfrak{A}_{h}\right) v=w$.

Moreover, the following are valid:

(ii) $\|v\|_{\infty} \leq\left(1-\lambda \alpha_{0}\right)^{-1}\left(1+\lambda e^{\alpha h}\right) r \leq r^{\prime}$;

(iii) $\left\|v_{1}-v_{2}\right\|_{1, r^{\prime}} \leq\left(1-\lambda \beta_{0}\right)^{-1}\left\|w_{1}-w_{2}\right\|_{1, r^{\prime}}$ if $\left(1-\lambda \mathfrak{A}_{h}\right) v_{j}=w_{j}$ and $\left\|w_{j}\right\|_{\infty} \leq r(j=1,2)$.

PROOF. First observe that the set $\left\{v \in L^{\infty}\left(R^{n}\right) ;\|v\|_{\infty} \leq r^{\prime}\right\}$ is closed under the norm $\|\cdot\|_{1, r^{\prime}}$. The equation $\left(1-\lambda \mathfrak{A}_{h}\right) v=w$ is rewritten as

$$
v=\frac{h}{h+\lambda} w+\frac{\lambda}{h+\lambda} S_{h} v
$$


Denoting the right-side by $\Psi_{\lambda}(v)$ we show the following.

$$
\left\|\Psi_{\lambda}(v)\right\|_{\infty} \leq r^{\prime} \quad \text { if }\|v\|_{\infty} \leq r^{\prime}
$$

and

$$
\left\|\Psi_{\lambda}\left(v_{1}\right)-\Psi_{\lambda}\left(v_{2}\right)\right\|_{1, r^{\prime}} \leq \theta(\lambda, h)\left\|v_{1}-v_{2}\right\|_{1, r^{\prime}}
$$

for $v_{j} \in L^{\infty}\left(R^{n}\right)$, with $\left\|v_{j}\right\|_{\infty} \leq r^{\prime}(j=1,2)$ and some $\theta(\lambda, h) \in(0,1)$. Assertion (i) then follows from the contraction mapping principle. By Lemma 2.1 we have

$$
\left\|\Psi_{\lambda}(v)\right\|_{\infty} \leq \frac{\lambda}{h+\lambda} e^{\alpha h} r^{\prime}+\frac{h}{h+\lambda}\left(\lambda e^{\alpha h}+1\right) r
$$

and

$$
\begin{aligned}
\left\|\Psi_{\lambda}\left(v_{1}\right)-\Psi_{\lambda}\left(v_{2}\right)\right\|_{1, r^{\prime}} & =\frac{\lambda}{h+\lambda}\left\|S_{h} v_{1}-S_{h} v_{2}\right\|_{1, r^{\prime}} \\
& \leq \frac{\lambda}{h+\lambda} e^{(\beta+\omega) h}\left\|v_{1}-v_{2}\right\|_{1, r^{\prime}} .
\end{aligned}
$$

Now the right-hand side of the first estimate does not exceed $r^{\prime}$ (by definition of $r^{\prime}$ ), which proves (4.2). Since $\beta_{0} \lambda<1$ we see that $\theta(\lambda, h)=\lambda(h+\lambda)^{-1} e^{(\beta+\omega) h} \in(0,1)$, so (4.3) is obtained. (ii) and (iii) are easily obtained by applying Lemma 2.1 to the equality $v=\Psi_{\lambda}(v)$.

As mentioned in the proof of Lemma 4.1(iii), the application of Lemma 2.1(iii) to (4.1) implies the inequality

$$
\left(1-\lambda \beta_{0}(h)\right)\left\|v_{1}-v_{2}\right\|_{1, r} \leq\left\|\left(\mathfrak{I}-\lambda \mathfrak{A}_{r}\right) v_{1}-\left(\mathfrak{I}-\lambda \mathfrak{A}_{h}\right) v_{2}\right\|_{1, r}
$$

for $v_{i}$ with $\left\|v_{i}\right\|_{\infty} \leq r$ and $r>0$. This means that for $h \in(0,1)$ and $\lambda \in\left(0, \lambda_{0}\right)$ the operator $\mathfrak{I}-\lambda \mathfrak{A}_{h}$ is injective on all of $L^{\infty}\left(R^{n}\right)$ and has an inverse operator $\left(\mathfrak{I}-\lambda \mathfrak{A}_{h}\right)^{-1}$. In what follows, we write

$$
\mathfrak{J}_{\lambda}(h)=\left(\mathfrak{I}-\lambda \mathfrak{A}_{h}\right)^{-1} \text { for } \lambda \in\left(0, \lambda_{0}\right) \text { and } h \in(0,1) .
$$

Hence the function $v$ defined by Lemma 4.1 may be written as $v=\mathfrak{J}_{\lambda}(h) w$.

In view of Lemma 4.1 we fix any $T>0$ and any $r>\|C\|_{\infty}$, and put

$$
R \geq r \cdot \max \left\{\exp \left[T\left(e^{\alpha}+2 \alpha_{0}(1)\right)\right], e^{\alpha T}(1+T)\right\} .
$$

Notice that

$$
r\left[\left(1-\lambda \alpha_{0}(h)\right)^{-1}\left(1+\lambda e^{\alpha h}\right)\right]^{k} \leq r \cdot \exp \left[k \lambda\left(e^{\alpha h}+2 \alpha_{0}(h)\right)\right] \leq R
$$

for $h \in(0,1), \lambda \in\left(0, \lambda_{0}\right)$ and $k \lambda \in[0, T]$, and

$$
e^{\alpha k h}\left(r+k h\|C\|_{\infty}\right) \leq e^{\alpha T}\left(r+T\|C\|_{\infty}\right) \leq R
$$

for $h \in(0,1)$ and $k h \in[0, T]$. Now Lemma 4.1(ii) states that $\mathfrak{J}_{\lambda}(h)^{k} w$ are well defined provided $h \in(0,1), \lambda \in\left(0, \lambda_{0}\right), k \lambda \in[0, T]$ and $\|w\|_{\infty} \leq r$. More precisely, we have the following.

LEMMA 4.2. For every $h \in(0,1), \lambda \in\left(0, \lambda_{0}\right)$ and every integer $k>0$ with $k \lambda \in[0, T]$, we have

$$
\left\|\mathfrak{J}_{\lambda}(h)^{k} w\right\|_{\infty} \leq r\left(1-\lambda \alpha_{0}(h)\right)^{-k}\left(1+\lambda e^{\alpha h}\right)^{k} \leq R .
$$

This lemma asserts that the set $\left\{u_{h}^{\lambda} ; \lambda \in\left(0, \lambda_{0}\right), h \in(0,1)\right\}$ constructed in $\S 3$ is bounded in $L^{\infty}\left(R^{n} \times(0, T)\right)$. Now fix $u_{0} \in \mathfrak{C}\left(R^{n}\right)$ with $\left\|u_{0}\right\|_{\infty} \leq r$ and set

$$
a_{j k}=\left\|\mathfrak{J}_{\mu}(h)^{j} u_{0}-\mathfrak{J}_{\lambda}(h)^{k} u_{0}\right\|_{1, R}
$$

where $h \in(0,1), 0<\mu \leq \lambda<\lambda_{0}(h), 0 \leq j \leq[T / \lambda]$ and $0 \leq k \leq[T / \mu]$. 
LEMMA 4.3. The following estimates hold:

(i)

(ii)

$$
\left(1-\lambda \beta_{0}\right)^{k+1} a_{0 k} \leq k \lambda\left\|\mathfrak{A}_{h} u_{0}\right\|_{1, R} \quad \text { for } 0 \leq k \leq[T / \lambda] .
$$

$$
\left(1-\mu \beta_{0}\right)^{j+1} a_{j 0} \leq j \mu\left\|\mathfrak{A}_{h} u_{0}\right\|_{1, R} \quad \text { for } 0 \leq j \leq[T / \mu] .
$$

Proof. Lemma 4.1(iii) gives

$$
\begin{aligned}
a_{0 k}= & \left\|u_{0}-\mathfrak{J}_{\lambda}(h)^{k} u_{0}\right\|_{1, R} \leq \sum_{l=0}^{k-1}\left\|\mathfrak{J}_{\lambda}(h)^{l} u_{0}-\mathfrak{J}_{\lambda}(h)^{l+1} u_{0}\right\|_{1, R} \\
& \leq\left\|u_{0}-\mathfrak{J}_{\lambda}(h) u_{0}\right\|_{1, R} \sum_{l=0}^{k-1}\left(1-\lambda \beta_{0}(h)\right)^{-l} \\
& \leq k\left\|u_{0}-\mathfrak{J}_{\lambda}(h) u_{0}\right\|_{1, R}\left(1-\lambda \beta_{0}(h)\right)^{-k},
\end{aligned}
$$

and

$$
\begin{aligned}
\left\|u_{0}-\mathfrak{J}_{\lambda}(h) u_{0}\right\|_{1, R} & =\left\|\mathfrak{J}_{\lambda}(h)\left(1-\lambda \mathfrak{A}_{h}\right) u_{0}-\mathfrak{J}_{\lambda}(h) u_{0}\right\|_{1, R} \\
& \leq\left(1-\lambda \beta_{0}(h)\right)^{-1} \lambda\left\|\mathfrak{A}_{h} u_{0}\right\|_{1, R} .
\end{aligned}
$$

This proves (i). (ii) is proved similarly.

LEMMA 4.4. For $0 \leq j \leq[T / \mu]$ and $0 \leq k \leq[T / \lambda]$ we have

$$
\begin{aligned}
& \left(1-\mu \beta_{0}\right)^{j+1}\left(1-\lambda \beta_{0}\right)^{k+1} a_{j k} \\
& \leq\left[\left((j \mu-k \lambda)^{2}+k \lambda^{2}\right)^{1 / 2}+\left((j \mu-k \lambda)^{2}+j \lambda \mu\right)^{1 / 2}\right]\left\|\mathfrak{A}_{h} u_{0}\right\|_{1, R} .
\end{aligned}
$$

ProOF. By Lemma 4.3 the assertion holds for $j=0$ or $k=0$. Set $\theta=\mu / \lambda$; then we have the resolvent equation:

$$
\mathfrak{J}_{\lambda}(h) w=\mathfrak{J}_{\mu}(h)\left[\theta w+(1-\theta) \mathfrak{J}_{\lambda}(h) w\right] .
$$

Hence, by Lemma 4.1(iii),

$$
\begin{aligned}
a_{j k}= & \left\|\mathfrak{J}_{\mu}(h)^{j} u_{0}-\mathfrak{J}_{\mu}(h)\left[\theta \mathfrak{J}_{\lambda}(h)^{k-1} u_{0}+(1-\theta) \mathfrak{J}_{\lambda}(h)^{k} u_{0}\right]\right\|_{1, R} \\
& \leq\left(1-\mu \beta_{0}\right)^{-1}\left\|\mathfrak{J}_{\lambda}(h)^{j-1} u_{0}-\theta \mathfrak{J}_{\lambda}(h)^{k-1} u_{0}-(1-\theta) \mathfrak{J}_{\lambda}(h)^{k} u_{0}\right\|_{1, R} \\
& \leq\left(1-\mu \beta_{0}\right)^{-1}\left(\theta a_{j-1, k-1}+(1-\theta) a_{j-1, k}\right) .
\end{aligned}
$$

The result is now obtained by induction on $j$ and $k$. See $[2, \S 1]$ for the detailed induction argument.

We are now in a position to prove Lemma 3.2. Since $u_{0} \in \mathfrak{C}\left(R^{n}\right)$, Lemma 2.7 shows that $\left\|\mathfrak{A}_{h} u_{0}\right\|_{1, R}$ is uniformly bounded in $h \in(0,1)$. Since $|t-\lambda[t / \lambda]| \leq \lambda$, Lemma 3.2 follows from Lemma 4.4 with $j=[t / \mu]$ and $k=[t / \lambda]$.

4.2. Proof of Lemma 3.3. Let $r, R>0$ be as in (4.4). For $v, w \in L^{\infty}\left(R^{n}\right)$ we define

$$
\begin{aligned}
{[v, w]_{-} } & =\sup _{t<0} t^{-1}\left(\|v+t w\|_{1, R}-\|v\|_{1, R}\right) \\
& =\lim _{t \downarrow 0} t^{-1}\left(\|v\|_{1, R}-\|v-t w\|_{1, R}\right) .
\end{aligned}
$$

The functional $[\cdot, \cdot]$ - may be regarded as the tangent functional of the unit sphere of the normed space $\left(L^{\infty}\left(R^{n}\right),\|\cdot\|_{1, R}\right)$ and, as is well known, it has the following properties:

$$
[v, a v]_{-}=a\|v\|_{1, R} \quad \text { for } a \in R^{1} ; \quad[v, a w]_{-}=a[v, w]_{-} \quad \text { for } a \geq 0 .
$$




$$
\begin{aligned}
{\left[v, w_{1}+w_{2}\right]_{-} } & \leq\left\|w_{1}\right\|_{1, R}+\left[v, w_{2}\right]_{-}, \\
{[v, a v+w]_{-} } & =a\|v\|_{1, R}+[v, w]_{-} \quad \text { for } a \in R^{1} .
\end{aligned}
$$

LEMMA 4.5. For $v, w \in L^{\infty}\left(R^{n}\right)$ with $\|v\|_{\infty},\|w\|_{\infty} \leq R$, we have

$$
\begin{aligned}
\left\|S_{h} v-w\right\|_{1, R} & \leq e^{(\beta+\omega) h}\|v-w\|_{1, R}+h\left[S_{h} v-w, \mathfrak{A}_{h} w\right]_{-} \\
& \leq e^{(\beta+\omega) h}\|v-w \mid\|_{1, R}+h\left\|\mathfrak{A}_{h} w\right\|_{1, R} .
\end{aligned}
$$

Proof. By (4.5) and (4.6),

$$
\begin{aligned}
\left\|S_{h} v-w\right\|_{1, R} & =\left[S_{h} v-w, S_{h} v-w\right]_{-}=\left[S_{h} v-w, S_{h} v-S_{h} w+S_{h} w-w\right]_{-} \\
& \leq\left\|S_{h} v-S_{h} w\right\|_{1, R}+h\left[S_{h} v-w, \mathfrak{A}_{h} w\right]_{-} .
\end{aligned}
$$

Applying (4.6) and Lemma 2.1(iii) to the right-hand side, we obtain the result.

This time, we take any $u_{0} \in \mathfrak{C}\left(R^{n}\right)$ with $\left\|u_{0}\right\|_{\infty} \leq r$ and set

$$
b_{k j}=\left\|S_{h}^{k} u_{0}-\mathfrak{J}_{\mu}(h)^{j} u_{0}\right\|_{1, R}
$$

for $0<\mu \leq h<1,0<\mu<\lambda_{0}(h), 0 \leq j \leq[T / \mu]$ and $0 \leq k \leq[T / h]$.

LEMMA 4.6. The following estimates hold:

$$
\begin{gathered}
e^{-(\beta+\omega) k h} b_{k 0} \leq k h\left\|\mathfrak{A}_{h} u_{0}\right\|_{1, R} \quad \text { for } 0 \leq k \leq[T / h] . \\
\left(1-\mu \beta_{0}\right)^{j+1} b_{0 j} \leq j \mu\left\|\mathfrak{A}_{h} u_{0}\right\|_{1, R} \quad \text { for } 0 \leq j \leq[T / \mu] .
\end{gathered}
$$

ProOF. Since (ii) was already proved in Lemma 4.3, it suffices to show (i). Putting $v=S_{h}^{k-1} u_{0}$ and $w=u_{0}$ in Lemma 4.5, we have

$$
b_{k 0} \leq e^{(\beta+\omega) h} b_{k-1,0}+h\left\|\mathfrak{A}_{h} u_{0}\right\|_{1, R} .
$$

From this the result follows by induction on $k$.

LEMMA 4.7. Let $\theta=(h+\mu)^{-1}$. Then

$$
\left\|S_{h} v-\mathfrak{J}_{\mu}(w)\right\|_{1, R} \leq e^{(\beta+\omega) h} \theta\left\|v-\mathfrak{J}_{\mu}(h) w\right\|_{1, R}+(1-\theta)\left\|S_{h} v-w\right\|_{1, R}
$$

for $v, w \in L^{\infty}\left(R^{n}\right)$ with $\|v\|_{\infty} \leq R$ and $\|w\|_{\infty} \leq R\left(1-\mu \alpha_{0}\right)\left(1+\mu e^{\alpha h}\right)^{-1}$.

ProOF. First observe that $\left\|\mathfrak{J}_{\mu}(h) w\right\|_{\infty} \leq R$. Hence Lemma 4.5 gives

$$
\left\|S_{h} v-J_{\mu}(h) w\right\|_{1, R} \leq e^{(\beta+\omega) h}\left\|v-\mathfrak{J}_{\mu}(h) w\right\|_{1, R}+h\left[S_{h} v-\mathfrak{J}_{\mu}(h) w, \mathfrak{A}_{h} \mathfrak{J}_{\mu}(h) w\right]_{-} .
$$

The last term is estimated as

$$
\begin{aligned}
h\left[S_{h} v-\mathfrak{J}_{\mu}(h) w, \mathfrak{A}_{h} \mathfrak{J}_{\mu}(h) w\right]_{-} & =(h / \mu)\left[S_{h} v-\mathfrak{J}_{\mu}(h) w, \mathfrak{J}_{\mu}(h) w-w\right]_{-} \\
& =(h / \mu)\left[S_{h} v-\mathfrak{J}_{\mu}(h) w, \mathfrak{J}_{\mu}(h) w-S_{h} v+S_{h} v-w\right]_{-} \\
& \leq-(h / \mu)\left\|S_{h} v-\mathfrak{J}(h) w\right\|_{1, R}+(h / \mu)\left\|S_{h} v-w\right\|_{1, R},
\end{aligned}
$$

from which the desired estimate is obtained.

We now prove Lemma 3.3. Putting $v=S_{h}^{k-1} u_{0}$ and $w=\mathfrak{J}(h)^{j-1} u_{0}$ in Lemma 4.7 , we have

$$
b_{k j} \leq e^{(\beta+\omega) h} \theta b_{k-1, j}+(1-\theta) b_{k, j-1},
$$

where $\theta=(h+\mu)^{-1} \mu$. An induction argument, together with Lemma 4.6, yields

$$
e^{-(\beta+\omega) k h}\left(1-\mu \beta_{0}\right)^{j+1} b_{k j} \leq\left[(k h-j \mu)^{2}+(k h+j \mu) h\right]^{1 / 2}\left\|\mathfrak{A}_{h} u_{0}\right\|_{1, R} .
$$


See $[13, \S 3]$ for the detailed induction argument. Since $u_{0} \in \mathfrak{C}\left(R^{n}\right)$, Lemma 2.7 shows that $\left\|\mathfrak{A}_{h} u_{0}\right\|_{1, R}$ is bounded uniformly for $h \in(0,1)$. Therefore, putting $k=[t / h], j=[t / \mu]$ in (4.7) and then using Lemma 3.2 , we conclude that the assertion of Lemma 3.3 is valid.

4.3. A product formula. The nonlinear semigroup $\{\mathfrak{S}(t): t \geq 0\}$ as mentioned in Theorem II is constructed in the following way.

THEOREM 4.8. There is a semigroup $\{\mathfrak{S}(t): t \geq 0\}$ of nonlinear operators on $L^{\infty}\left(R^{n}\right)$ with the following properties:

(i) For each $v \in L^{\infty}\left(R^{n}\right)$ we have the product formula

$$
\mathfrak{S}(t) v=\lim _{h \downarrow 0} S_{h}^{[t / h]} v \quad \text { in } L_{\text {loc }}^{1}\left(R^{n}\right),
$$

where the convergence holds uniformly for bounded $t$.

(ii)

$$
\|\mathfrak{S}(t) v\|_{\infty} \leq e^{\alpha t}\left(\|v\|_{\infty}+t\|C\|_{\infty}\right) \quad \text { for } t \geq 0 \text { and } v \in L^{\infty}\left(R^{n}\right),
$$

where $C$ is the function defined in (H.2).

(iii) For each $r \geq\|C\|_{\infty}, T>0$ and each $R \geq r e^{\alpha T}(1+T)$, we have

$$
\|\mathfrak{S}(t) v-\mathfrak{S}(t) w\|_{1, R} \leq e^{(\beta+\omega) t}\|v-w\|_{1, R}
$$

for $t \in[0, T]$ and $v, w \in L^{\infty}\left(R^{n}\right)$ with $\|v\|_{\infty} \leq r,\|w\|_{\infty} \leq r$.

(iv) Let $\leq$ be the natural order relation in $L_{\text {loc }}^{1}\left(R^{n}\right)$. Then each $\mathfrak{S}(t)$ is orderpreserving in the sense that if $v, w \in L^{\infty}\left(R^{n}\right)$ and $v \leq w$ then $\mathfrak{S}(t) v \leq \mathfrak{S}(t) w$.

(v) For each $v \in L^{\infty}\left(R^{n}\right)$ the function $u(x, t)=[\mathfrak{S}(t) v](x)$ gives an entropy solution of $(\mathrm{M})$ with the initial value $v$.

ProOF. Given a $v \in L^{\infty}\left(R^{n}\right)$, let $u(t ; v) \equiv u(\cdot, t ; v)$ be the $L^{\infty}\left(R^{n}\right)$-valued function on $[0, \infty)$ which is obtained through the convergence $(1.2)$ (with $u_{0}$ replaced by $v$ ). Then one can define, for each $t \geq 0$, an operator $\mathfrak{S}(t): L^{\infty}\left(R^{n}\right) \rightarrow L^{\infty}\left(R^{n}\right)$ by setting

$$
\mathfrak{S}(t) v=u(t ; v) \text { for } v \in L^{\infty}\left(R^{n}\right) .
$$

We shall show that the family of operators $\{\mathfrak{S}(t): t \geq 0\}$ gives the desired semigroup. Firstly, Proposition 3.1(iii), together with the estimate (3.17), states that for each $v \in L^{\infty}\left(R^{n}\right)$ the mapping $t \rightarrow \mathfrak{S}(t) v$ is continuous from $[0, \infty)$ into $L_{\text {loc }}^{1}\left(R^{n}\right)$ and $\mathfrak{S}(0) v=v$. Hence $\{\mathfrak{S}(t)\}$ satisfies the continuity condition (1.5). Secondly, it was shown with the aid of Lemmas 3.2 and 3.3 that for each $v \in L^{\infty}\left(R^{n}\right)$ the function $u(x, t) \equiv[\mathfrak{S}(t) v](x)$ gives a unique entropy solution of (M) with initial value $v$. (In fact, the convergence (1.2) itself was obtained through the uniqueness of entropy solutions.) Now the semigroup property $(1.3)$ of $\{\mathfrak{S}(t)\}$ follows from this fact. Thirdly, combining (4.8), the definition (1.1) of approximate solutions and Proposition 2.8, we see that the operators $\mathfrak{S}(t)$ are represented by the product formula

$$
\mathfrak{S}(t) v=\lim _{h \downarrow 0} S_{h}^{[t / h]} v \quad \text { in } L_{\text {loc }}^{1}\left(R^{n}\right),
$$

where the convergence is uniform for bounded $t$. All properties of $\{\mathfrak{S}(t)\}$ in (ii)(iv) are derived from (4.9): (ii) follows from Proposition 2.2(i) and (4.9), (iii) is a 
consequence of Proposition 2.2(iii) and (4.9), and (iv) follows from (4.9) and the fact that the operators $S_{h}, h>0$, are all order-preserving (see the proof of Lemma 2.1). In particular, (iii) states that $\{\mathfrak{S}(t)\}$ satisfies condition (1.4) since for each pair $r, R$ with $r \leq R$, the relative topology (induced by the metric topology of $L_{\text {loc }}^{1}\left(R^{n}\right)$ ) on the closed convex subset $X_{r} \equiv\left\{v \in L^{\infty}\left(R^{n}\right):\|v\|_{\infty} \leq r\right\}$ is equivalent to the metric topology on $X_{r}$ defined by the metric $d_{R}(v, w)=\|v-w\|_{1, R}, v, w \in X_{r}$. Thus $\{\mathfrak{S}(t)\}$ forms a nonlinear semigroup on $L^{\infty}\left(R^{n}\right)$ with the properties listed in (i) $-(\mathrm{v})$, and the proof is complete.

Finally we give a few remarks on the results mentioned above.

(1) It would be interesting to discuss the "generator" of the semigroup $\{\mathfrak{S}(t)\}$. The detailed argument concerning this problem will be given elsewhere.

(2) It is seen from the proof of Lemma 3.2 that for each $h>0$, the operator $\mathfrak{A}_{h}$ generates a semigroup $\left\{\mathfrak{S}_{h}(t): t \geq 0\right\}$ of nonlinear operators on $L^{\infty}\left(R^{n}\right)$ in the sense that the exponential formula

$$
\mathfrak{S}_{h}(t) v=\lim _{\lambda \downarrow 0}\left(I-\lambda \mathfrak{A}_{h}\right)^{-[t / \lambda]} v \quad \text { in } L_{\text {loc }}^{1}\left(R^{n}\right)
$$

holds for $v \in L^{\infty}\left(R^{n}\right)$ and $t \geq 0$. Theorem 4.8(i) can be regarded as an approximation theorem for the semigroup $\{\mathfrak{S}(t)\}$. Now using the semigroups $\left\{\mathfrak{S}_{h}(t)\right\}$, $h \in(0,1)$, we have another approximation theorem for $\{\mathfrak{S}(t)\}$.

Proposition 4.9. For each $v \in L^{\infty}\left(R^{n}\right)$,

$$
\mathfrak{S}(t) v=\lim _{h \downarrow 0} \mathfrak{S}_{h}(t) v \quad \text { in } L_{\mathrm{loc}}^{1}\left(R^{n}\right)
$$

where the convergence is uniform for bounded $t$.

\section{REFERENCES}

1. H. Brezis and A. Pazy, Convergence and approximation of nonlinear semigroups in Banach spaces, J. Funct. Anal. 9 (1972), 63-74.

2. M. G. Crandall and T. M. Liggett, Generation of semi-groups of nonlinear transformations on general Banach spaces, Amer. J. Math. 93 (1971), 265-298.

3. M. G. Crandall, The semigroup approach to first order quasilinear equations in several space variables, Israel J. Math. 12 (1972), 108-132.

4. M. G. Crandall and A. Majda, Monotone difference approximations for scalar conservation laws, Math. Comp. 34 (1980), 1-21.

5. Y. Giga and T. Miyakawa, A kinetic construction of global solutions of first order quasilinear equations, Duke Math. J. 50 (1983), 505-515.

6. E. Giusti, Minimal surfaces and functions of bounded variation, Notes on Pure Math., no. 10, Australian National Univ., Canberra, 1977.

7. A. Harten, P. D. Lax and B. van Leer, On upstream differencing and Godunov-type schemes for hyperbolic conservation laws, SIAM Rev. 25 (1983), 35-61.

8. Y. Kobayashi, The application of the product formula for semigroups to first onder quasilinear equations, Hiroshima Math. J. (to appear).

9. K. Kobayasi and S. Oharu, On nonlinear evolution operators associated with certain nonlinear equations of evolution, Lecture Notes Numer. Appl. Math. 2 (1980), 139-210.

10. S. N. Kružkov, First onder quasilinear equations in several independent variables, Math. USSR-Sb. 10 (1970), 217-243.

11. P. D. Lax, Hyperbolic systems of conservation laws and the mathematical theory of shock waves, CBMS Regional Conf. Ser. Appl. Math., no. 11, SIAM, Philadelphia, Pa., 1973.

12. W. Mazja, Einbettungssätze für Sobolewsche Räume, Teil 2, Teubner, Leipzig, 1980. 
13. I. Miyadera and Y. Kobayashi, Convergence and approximation of nonlinear semigroups, Functional Analysis and Numerical Analysis, Japan-France Seminar, Tokyo and Kyoto (H. Fujita, ed.), 1976.

14. S. Oharu and T. Takahashi, A convergence theorem of nonlinear semigroups and its application to first order quasilinear equations, J. Math. Soc. Japan 26 (1974), 126-160.

15. O. A. OleInik, Discontinuous solutions of non-linear differential equations, Amer. Math. Soc. Transl. (2) 26 (1963), 95-172.

16. Y. Brenier, Une application de la symétrisation de Steiner aux équations hyperboliques: la méthode de transport et écroulement, C. R. Acad. Sci. Paris 292 (1981), 563-566.

17. __ Résolution d'équations d'évolution quasilinéaries en dimension $N$ d'espace à l'aide d'équations linéaires en dimension $N+1$, J. Differential Equations 49 (1983).

18. T. Miyakawa, A kinetic approximation of entropy solutions of first order quasilinear equations, Lecture Notes Numer. Appl. Anal. 6 (1983), 93-105.

Department of MAThematics, NAgoya University, NAgoya 464, JAPAN (Current address of Y. Giga)

Department of MAThematics, HiRoshima University, Hiroshima 730, Japan (Current address of T. Miyakawa and S. Oharu) 